\title{
A PROSPECTIVE STUDY COMPARING STENTING VERSUS NON-STENTING AFTER UNCOMPLICATED URETEROSCOPY FOR URETERIC STONES
}

\author{
Sreedhar Reddy¹, Prathvi², Mayank Kulshreshtha ${ }^{3}$, Padmakar Singh ${ }^{4}$ \\ 1 Professor and HOD, Department of Urology, Raja Rajeshwari Medical College and Hospital, Bangalore. \\ ${ }^{2}$ Assistant Professor, Department of Urology, Raja Rajeshwari Medical College and Hospital, Bangalore. \\ 3 Post Graduate, Department of Urology, Raja Rajeshwari Medical College and Hospital, Bangalore. \\ 4 Post Graduate, Department of Urology, Raja Rajeshwari Medical College and Hospital, Bangalore.
}

\begin{abstract}
The placement of stents has been a standard practice since 1967. The advantage of routine stenting is that it minimizes postoperative ureteral obstruction and renal colic that may result from ureteral oedema caused by balloon dilatation or stone manipulation. There are various disadvantages resulting from it including flank pain, voiding symptoms, infections, stent related stone formation and encrustation. Various studies recommend them to be used only for procedures with complications such as ureteric injury or if a stone fragment remained at the end of the procedure. The aim of our study was to assess the need for routine ureteral stenting after uncomplicated ureteroscopic stone removal.
\end{abstract}

\section{MATERIALS AND METHODS}

This study was conducted at Rajarajeshwari Medical College and Hospital, Bangalore, as a prospective randomized controlled trial in Department of Urology between January 2015 and May 2016. In this hospital-based prospective, comparative study, all patients were treated by ureteroscopic lithotripsy. Following the procedure, patients were randomized to the non-stented ( $\mathrm{n}=44$ ) who had no stent placed at the end of the operation and stented $(n=52)$ group having Double DJ stent placement. The assessment criteria included operative time, success rate, postoperative analgesia, complications including (Flank pain, dysuria, haematuria, frequency/urgency), UTI, fever, rehospitalisation and hospital stay.

\section{RESULTS}

The two groups were comparable with respect to baseline variables of age, gender, mean stone size, side of stone and number of patients turning for radiological follow-up at 3 months. The mean operative time was $38.12 \pm 10.76$ minutes in the stented group and $32.22 \pm 6.72$ minutes in the non-stented group. However, this difference was statistically insignificant. There was no significant difference in the two treatment groups with regard to use of intracorporeal lithotripsy. Ureteral dilatation was not required in any patient in both groups. A successful outcome was achieved in $100 \%$ of the cases in both groups. Patients with double J stents had statistically significantly more frequency/urgency, dysuria and need of analgesics compared to those without stents. There was no significant difference between the three groups regarding haematuria, fever, flank pain, urinary tract infection, rehospitalisation and mean hospital stay.

\section{CONCLUSION}

Routine ureteral stenting does not appear to be warranted in those patients who do not require ureteral dilation during ureteroscopic procedures. Patients without stents had significantly less pain, fewer urinary symptoms and decreased analgesic use postoperatively. Another advantage is cost effectiveness and avoidance of cystoscopy for stent removal.

\section{KEYWORDS}

Stent, Ureteroscopy, Lithotripsy, Ureteral Dilatation.

HOW TO CITE THIS ARTICLE: Reddy S, Prathvi, Kulshreshtha M, et al. A prospective study comparing stenting versus non-stenting after uncomplicated ureteroscopy for ureteric stones. J. Evolution Med. Dent. Sci. 2016;5(61):4291-4294, DOI: $10.14260 /$ jemds/2016/979

\section{INTRODUCTION}

First ureteroscopy was performed by Hugh Hampton Young in 1912 on a patient with posterior urethral valve.(1) In 1983 Huffman performed first ureteroscopic removal of ureteric stone.(2) Among various treatment modes for ureteral stone, ureteroscopy is one of the options. Decrease in the size of the ureteroscope and the use of flexible variety has made the procedure of stone removal more effective.

Financial or Other, Competing Interest: None.

Submission 04-07-2016, Peer Review 21-07-2016,

Acceptance 23-07-2016, Published 01-08-2016.

Corresponding Author:

Dr. Sreedhar Reddy,

Professor and HOD,

Department of Urology,

Raja Rajeshwari Medical College and

Hospital, Bangalore.

E-mail: urologyreddy@yahoo.com

DOI: $10.14260 /$ jemds $/ 2016 / 979$
The placement of stents has been a standard practice since 1967.(3) The advantage of routine stenting is that it minimises postoperative ureteral obstruction and renal colic that may result from ureteral oedema caused by balloon dilatation or stone manipulation.(4) Routine stenting has also been thought to promote healing and reduce the incidence of ureteral stricture.(5)

Though ureteral stents have been an indispensable tool, their use is now being questioned. There are various disadvantages resulting from it including flank pain, voiding symptoms, infections, stent related stone formation and encrustation. (6,7) Thus, various studies recommend them to be used only for procedures with complications such as ureteric injury or if a stone fragment remained at the end of the procedure. $(8,9)$

The aim of our study was to assess the need for routine ureteral stenting after uncomplicated ureteroscopic stone 
removal, evaluating the patient characteristics, stone features, complications and treatment outcome among stented and unstented patients.

\section{MATERIALS AND METHODS}

This study was conducted at Rajarajeshwari Medical College and Hospital, Bangalore, after receiving clearance from Ethical Committee. This study was designed as a prospective randomized controlled trial conducted in Department of Urology between January 2015 and May 2016.

\section{Inclusion Criteria}

1. Stones sized less than $15 \mathrm{~mm}$.

2. Absence of complications during the procedure including ureteric injury, evidence of mucosal oedema or haemorrhage.

\section{Exclusion Criteria}

1. Patients with history of sepsis or renal failure.

2. Patients with bilateral ureteric stones.

3. Patient with a solitary kidney.

4. Patients who had an indwelling ureteral stent at the time of ureteroscopy.

5. Stones bigger than $15 \mathrm{~mm}$.

6. Stone present in pelvis.

7. Any complications after the procedure.

8. Patients not fit for anaesthesia.

9. Pregnant females.

All of the patients underwent URSL by intraluminal Pneumatic (ballistic) Swiss LithoClast with semi-rigid Storz ureteroscope of 6-fr under spinal anaesthesia and were catheterized post procedure and kept in post-operative ward. All of the patients were given IV fluid, analgesia and proton pump blocker for 6 hours, then switched to oral medication. Patients were randomized into stented and not stented group depending on the procedure. Successful ureteroscopy was defined as complete extraction or fragmentation of the stone. Foley catheter was left indwelling till the next morning in order to avoid mobilization after spinal anaesthesia, so that the patient could be spared voiding problems.

All patients were checked on 1st postoperative day with Xray KUB for radiological clearance of stone and position of Double-J stent. Patients were discharged on first or second postoperative day, and a follow-up visit was scheduled at 3 weeks, at which time patients in the stented group underwent stent removal under local anaesthesia. Age, gender, stone characteristics, total operating time, the mean operative time and mean hospital stay were all recorded. For postoperative symptoms and the complications, a special questionnaire and a precise clinical exam were used, looking for flank pain, haematuria, dysuria, urgency, fever and urinary tract infection. Postoperative pain and dysuria were measured using a $10 \mathrm{~cm}$ visual analog scale. We also studied the need of analgesics in postoperative period and the rate of rehospitalisation. Patients were advised to make further visits at 3-month intervals to detect any complication. They were advised to use oral analgesics for any pain or discomfort in the intervening period.

Statistical analysis was performed using Statistical Package for Social Sciences software (SPSS) version 16.

\section{RESULTS}

A total of 96 patients undergoing ureteroscopy for ureteric stones completed the study protocol with 52 patients in the stented group and 44 patients in the non-stented group. The two groups were comparable with respect to baseline variables of age, gender, mean stone size, side of stone and number of patients turning for radiological follow-up at 3 months (Table 1).

\begin{tabular}{|c|c|c|c|}
\hline & $\begin{array}{c}\text { Stent } \\
(n=52)\end{array}$ & $\begin{array}{c}\text { No Stent } \\
(n=44)\end{array}$ & $\begin{array}{c}\text { P- } \\
\text { value }\end{array}$ \\
\hline $\begin{array}{l}\text { Mean Age } \pm \text { S.D. } \\
\text { (in Years) }\end{array}$ & $35.38 \pm 9.84$ & $31.53 \pm 8.31$ & n.s \\
\hline Gender M/F & $36 / 16$ & $30 / 14$ & n.s \\
\hline $\begin{array}{c}\text { Mean Stone Size } \\
\pm \text { S.D. (In mm) }\end{array}$ & $7.22 \pm 1.24$ & $7.08 \pm 1.32$ & n.s \\
\hline $\begin{array}{l}\text { Radiological Follow } \\
\text { Up (\%) (3 Months) }\end{array}$ & $\begin{array}{l}\text { 41/52 pts } \\
(78.84 \%)\end{array}$ & $\begin{array}{l}34 / 44 \mathrm{pts} \\
(77.27 \%)\end{array}$ & n.s \\
\hline Side of Stone R/L & $20 / 32$ & $18 / 26$ & n.s \\
\hline
\end{tabular}

\begin{tabular}{|c|c|c|c|}
\hline & $\begin{array}{c}\text { Stent } \\
(\mathbf{n = 5 2})\end{array}$ & $\begin{array}{c}\text { No Stent } \\
(\mathbf{n = 4 4 )}\end{array}$ & $\begin{array}{c}\text { P } \\
\text { value }\end{array}$ \\
\hline $\begin{array}{c}\text { Ureteral } \\
\text { dilatation }\end{array}$ & none & none & - \\
\hline Lithotripsy (\%) & $38(73.07)$ & $35(79.54)$ & n.s \\
\hline Success (\%) & $52(100)$ & $44(100)$ & - \\
\hline $\begin{array}{c}\text { Mean Operative } \\
\text { Time } \pm \text { S.D. (min) }\end{array}$ & $38.12 \pm 10.76$ & $32.22 \pm 6.72$ & n.s \\
\hline \multicolumn{2}{|c|}{ Table 2: Operative Characteristics } \\
\hline
\end{tabular}

The mean operative time was $38.12 \pm 10.76$ minutes in the stented group and $32.22 \pm 6.72$ minutes in the non-stented group (Table 2). Although in the non-stented group time was less, but this difference was statistically insignificant. There was no significant difference in the two treatment groups with regard to use of intracorporeal lithotripsy. Ureteral dilatation was not required on any patient in both groups. A successful outcome was achieved in $100 \%$ of the cases in both groups.

\begin{tabular}{|c|c|c|c|}
\hline & $\begin{array}{c}\text { Stent } \\
(\mathbf{n = 5 2 )}\end{array}$ & $\begin{array}{c}\text { No Stent } \\
(\mathbf{n = 4 4 )}\end{array}$ & $\begin{array}{c}\text { P- } \\
\text { value }\end{array}$ \\
\hline $\begin{array}{c}\text { Mean Flank Pain Score at } \\
48 \text { hrs. (0-10) } \pm \text { S.D. }\end{array}$ & $4.4 \pm 2.1$ & $4.8 \pm 2.0$ & $n . s$ \\
\hline Dysuria Number (\%) & $14(26.93)$ & $6(13.64)$ & $n . s$ \\
\hline Haematuria Number (\%) & $15(28.84)$ & $10(22.73)$ & $n . s$ \\
\hline $\begin{array}{c}\text { Frequency/Urgency } \\
\text { Number (\%) }\end{array}$ & $25(48.07)$ & $8(18.18)$ & $<0.001$ \\
\hline $\begin{array}{c}\text { Need for Analgesics in } \\
\text { Follow-Up }\end{array}$ & $15(28.84)$ & $4(9.09)$ & $<0.001$ \\
\hline Fever (\%) & $7(13.46)$ & $6(13.64 \%)$ & n.s \\
\hline UTI (\%) & $7(13.46)$ & $6(13.64 \%)$ & n.s \\
\hline $\begin{array}{c}\text { Re-Hospitalisation } \\
\text { Number }\end{array}$ & 1 & 0 & $n . s$ \\
\hline $\begin{array}{c}\text { Mean Hospital } \\
\text { Stay (Hours) }\end{array}$ & 52 hrs. & 54 hrs. & n.s \\
\hline \multicolumn{3}{|c|}{ Table 3: Post-Operative Symptoms and Complications } \\
\hline
\end{tabular}

Table 3 shows the mean visual analog pain scores at 48 hours and other postoperative symptoms and complications in both groups. Patients with double-J stents had statistically 
significantly more frequency/urgency, dysuria, and need of analgesics compared to those without stents. There was no significant difference between the three groups regarding haematuria, fever, flank pain, urinary tract infection, rehospitalisation and mean hospital stay.

One patient in the stented group developed urinary sepsis with urine culture showing growth of E. coli sp., necessitating treatment with intravenous antibiotics and prolonging the hospital stay.

\section{DISCUSSION}

Ureteral stents are commonly placed after ureteroscopic stone extraction. The rationale for the routine use of ureteral stents after ureteroscopy originates from supposition rather than from evidence-based medicine. Though the use of DJ stent after the stone extraction was previously advocated by the urologists after all ureteroscopic lithotripsy, its routine use is currently debatable. However, the placement of a ureteral stent may be associated with significant morbidity.

Joshi et al identified patient morbidity associated with ureteral stents as a significant health problem.(10) They found that ureteral stents are associated with significant symptoms, such as pain affecting daily activities (80\%), urinary symptoms (73\%), and reduced work capacity (58\%), which reduce quality of life. Stents profoundly affect physical and psychosocial health and have a negative impact on functional capacity and work performance.(10,11)

Indwelling ureteral stents may be associated with significant symptoms and signs such as pain, urgency, dysuria, and haematuria and may lead to complications such as stent, migration and urosepsis.(12-14) Pollard and MacFarlane reported that $90 \%$ of their patients who had indwelling stents had one or more upper or lower urinary-tract symptoms. (14) Bregg and Richle reported that $44 \%$ of the patients who had stents placed before SWL had moderate-to-intolerable discomfort that was relieved by removal of the stents.(13) Routine placement of an indwelling stent also adds to the overall cost of the procedure and necessitates additional cystoscopy for stent removal unless a stent with a pull string is used. It is obvious that if a stent could be avoided in some patients, it would greatly improve the postoperative course, reduce the cost of the procedure, and save valuable operating room time.

Several trials have demonstrated similar postoperative complication rates among stented and unstented patient populations. These trials have several similarities with our study including a preponderance of patients with distal ureteral calculi, uncomplicated procedure and absence of ureteral dilation.

In the study of Ibrahim et al, early postoperative complications including low-grade fever, haematuria and urinary tract infection were observed in $20 \%$ patients in nonstented patients and $19 \%$ patients in stented ones, a difference of no significant value. Mean initial hospitalization and time to return to normal physical activity were not different between the 2 groups. At 48 hours, there was no significant difference in flank pain between the 2 groups.(15)

Nabi et al reported the results of a meta-analysis of 9 randomized, controlled trials of stenting following uncomplicated ureteroscopy. The incidence of lower urinary tract symptoms was significantly higher in participants who had a stent inserted after ureteroscopy.(16)
In a recent meta-analysis studying, the effect of ureteral stent placement on post ureteroscopic complications, the authors concluded that the published evidence supports the practice of omitting an ureteral stent after an uncomplicated ureteroscopic procedure.(17)

Richter et al claimed that placement of a ureteral stent is "a friendly procedure with unfriendly high morbidity." The additional cystoscopy for stent removal is a cause of discomfort and overall cost.(18)

During the first three weeks of post-operative period, the stented group had more consumption of oral analgesics in comparison to the non-stented group. Similar findings have been revealed in various other studies.(19-21)

Present study has certain shortcomings. Ureteroscopy was not performed by a single urologist, which may have influenced the outcome. Similarly, the postoperative pain relief protocol was not standardized, being left to the discretion of the operating surgeon (On an as-needed basis or regularly timed medication).

\section{CONCLUSION}

Routine ureteral stenting does not appear to be warranted in those patients who do not require ureteral dilation during ureteroscopic procedures. Patients without stents had significantly less pain, fewer urinary symptoms and decreased analgesic use postoperatively. Another advantage is cost effectiveness and avoidance of cystoscopy for stent removal. Long-term complications like development of ureteric strictures among the stented and non-stented needs further evaluation.

\section{REFERENCES}

1. Agrawal A, Shrestha P, Belokar WK. Early experience in ureteroscopy for the management of ureteric stone. JCMSNepal 2011;7(4):28-33.

2. Prabhakar M. Retrograde ureteroscopic intrarenal surgery for large (1.6-3.5cms) upper ureteric/renal calculus. Indian J Urol 2010;26(1):46-9.

3. Miyaoka R, Monga M. Ureteral stent discomfort: aetiology and management. Indian J Urol 2009;25(4):455-60.

4. Boddy SM, Nimmon CC, Jones S, et al. Acute ureteric dilatation after ureteroscopy: an experimental study. Br J Urol 1988;61(1):27-31.

5. Harmon WJ, Sershon PD, Blute ML, et al. Ureteroscopy: current practice and long term complications. J Urol 1997;157(1):28-32.

6. Papadovkakis S, Stolzenburg JU, Truss MC. Treatment strategies of ureteral stones. EAU-EBU update series 2006;4:184-90.

7. Ahallal Y, Khallouk A, Farih MH. Risk factors analysis of management of ureteral double-J stent complications. Rev Urol 2010;12(2-3):e147-51.

8. Hubner WA, Plas EG, Stoller ML. The double J ureteral stent: in vivo and in vitro flow studies. J Urol 1992;148(2Pt 1):278-80.

9. Ullah I, Alam K, Wazir BG, et al. Indications and morbidity of indwelling ureteral stenting. Ann Pak Inst Med Sci 2011;7(4):173-5.

10. Joshi HB, Stainthorpe A, MacDonagh RP, et al. Indwelling ureteral stents: evaluation of symptoms, quality of life and utility. Journal of Urology 2003;169(3):1065-9. 
11. Joshi HB, Newns N, Stainthorpe A, et al. Ureteral stent symptom questionnaire: development and validation of a multidimensional quality of life measure. Journal of Urology 2003;169(3):1060-4.

12. Pollard SG, Macfarlane R. Symptoms arising from doubleJ ureteral stents. J Urol 1988;139(1):37-8.

13. Bregg K, Riehle RA. Morbidity associated with indwelling internal ureteral stents after shock wave lithotripsy. J Urol 1989;141(3):510-2.

14. Pryor JL, Langley MJ, Jenkins AD. Comparison of symptom characteristics of indwelling ureteral catheters. J Urol 1991;145(4):719-22.

15. Ibrahim HM, Al-Kandari AM, Shaaban HS, et al. Role of ureteral stenting after uncomplicated ureteroscopy for distal ureteral stones: a randomized, controlled trial. Journal of Urology 2008;180(3):961-5.

16. Nabi G, Cook J, N'Dow J, et al. Outcomes of stenting after uncomplicated ureteroscopy: systematic review and meta-analysis. British Medical Journal 2007; 334(7593):572-5.
17. Makarov DV, Trock BJ, Allaf ME, et al. The effect of ureteral stent placement on post-ureteroscopy complications: a meta-analysis. Urology 2008;71(5):796-800.

18. Richter S, Ringel A, Shalev M, et al. The indwelling ureteric stent: a friendly procedure with unfriendly high morbidity. BJU International 2000;85(4):408-11.

19. Chen JJ, Yip SKH, Wong MY, et al. Ureteroscopy as an outpatient procedure: the Singapore general hospital urology centre experience. Hong Kong Med J 2003;9(3):175-8.

20. Alapont JM, Broseta E, Oliver F, et al. Ureteral avulsion as a complication of ureteroscopy. Int Braz J Urol 2003;29(1):18-22.

21. Chen YT, Chen J, Wong WY, et al. Is ureteric stenting necessary after uncomplicated ureteroscopic lithotripsy? A prospective randomized controlled trial. J Urol 2002;167(5):1977-80. 